\title{
Relativistic Electromagnetic-Gravitational Interaction
}

\section{Vegt JM*}

Technical University Eindhoven, Netherlands

\begin{abstract}
A change in approach in the theory of Relativistic Electromagnetic Fields, introduced by James Clerk Maxwel in 1865 and the theory of "Electric-Magnetic/Space-Time" conversions by Einstein-Lorentz-Minkovski in 1905 in the theory of Special Relativity (specially described by the Lorentz-Transformations) and the Theory of General Relativity (GTR) by Albert Einstein in 1915 has been presented in this article.

What is known in Astrophysics has originally been based on the fundamental knowledge of the classical Electromagnetic theories of James Clerk Maxwell and the famous Theory of General Relativity by Albert Einstein. Electromagnetic radiation and in specific light has always been the carrier for this information. That is a fundamental problem. Because what do we really know about light? When James Maxwell published his famous set of 4 electromagnetic equations, the Maxwell equations, he did not built his theory on a logical fundament. His foundation was not logical at all. Why would a set of 4 electromagnetic equations which are correct at low frequencies describe a physical phenomenon like the propagation of light with a speed that at that time had never been measured before? Since then nobody ever doubted about the correctness of the theories of Maxwell. The success of his predictions made Maxwell famous. But fame is not a ground for fundamental science.
\end{abstract}

Keywords: Electromagnetic equations; General relativity; Newton's law

\section{Introduction}

The famous eclipse expedition of 1919 to Sobral, Brazil, and the island of Principe, in the Gulf of Guinea, led by Dyson, Eddington and Davidson was a turning point in the acceptance of the General Theory of Relativity developed by Albert Einstein in 1915. The correctness of this theory has been proven since then in many experiments. The success of his predictions made Einstein famous [1-5]. But fame is not a ground for real science. Because what do we really know about light? When Albert Einstein published his famous Einstein Field Equations (Einstein-Maxwell equations), which were the mathematical foundation on which the Theory of General Relativity has been built, he did not built his theory on a logical fundament. Einstein built his theory on the complex 4-dimensional Minkovski Space, developed by Hermann Minkovski, the Electromagnetic Lorentz Transformations, developed by Hendrik Antoon Lorentz and the fundamental Maxwell Equations. His foundation was not logical at all but grounded on the success of those who became famous before him. But fame is never a ground for fundamental science. Einstein had a great imagination and was inspired by the imaginary 4-dimensional complex space-time continuum, suggested by his teacher Hermann Minkovski. But his foundation was not logical at all. Why would space and time be equal. We do experience space and time always very differently [6-13]. When we shine the light into a room, all three spatial dimensions of the room will be enlightened and become visible. But the fourth dimension time will always remain invisible. We cannot look into the future and we cannot look back in time by the illumination of light that shines into time. There is no logical ground for the Theory of General Relativity. Since the success of the predictions of Albert Einstein, nobody ever doubted about the correctness of the theories of Albert Einstein. The success of his predictions made Einstein famous.

In this manuscript, the starting point is almost 300 years ago. Long before James Maxwell published his Maxwell equations, before Albert Einstein published the Theory of General Relativity and far before the time Niels Bohr introduced the probability waves, discovered by Erwin Schrödinger which were the nonlogical grounds on which Quantum Physics has been built.
The starting point is the moment just after Isaac Newton had published the breaking of light into the colors of the rainbow through a prism in the "Lectiones Opticae" in 1728. Newton just had discovered the beauty of light when light had revealed one of its many secrets. And he could only express: "Light is beautiful".

That has been the turning point in our history of science. Since then science has left the path of the light and has followed the path of our own mind. We did not follow anymore, but we started to lead. To lead in arrogance and surrounded by the illusions of fame. But instead of following the path of the mind that has left every logical thinking, a path that is finally following the path of quantum physics and elementary particles, the ghost lights that will lead us to the swamps of darkness, this manuscript wants to return to the path of the light. Because the light has always given the right answers. And has always guided mankind in science and through life. What would our world be without the beauty of light? When we look at the fruits of our modern science we can only see the fruits of darkness. A darkness where the predators are hiding to destroy our world of beauty, our world of light, our world of truth. Destroying this world of beauty by lies, by ghost lights of the swamps of darkness. Who are we following? The gambling devil leading us into the swamps of darkness? The fake light of the illusion of fame that will lead us into the deepest darkness the world has ever known?

When we return back to the world of light, we simply have to ask one question to the light. How is it possible that the light in our world does exist? And we simply follow Newton's third law of equilibrium published in the "Principia Mathematica Philisophiae Naturalis" in $1686[14]$

*Corresponding author: Vegt JM, Professor, Technical University Eindhoven Netherlands, Tel: 652265366; E-mail: jvegt@planet.nl

Received Mar 13, 2018; Accepted April 30, 2018; Published May 08, 2018

Citation: Vegt JM (2018) Relativistic Electromagnetic-Gravitational Interaction. J Phys Math 9: 268. doi: 10.4172/2090-0902.1000268

Copyright: (c) 2018 Vegt JM. This is an open-access article distributed under the terms of the Creative Commons Attribution License, which permits unrestricted use, distribution, and reproduction in any medium, provided the original author and source are credited. 
This single fundamental only question has been asked: What are the fundamental boundaries which are required for a stable electromagnetic field configuration? And instead of taking the 4 well-known electromagnetic low frequency equations and put them together and find exactly the same results as James Maxwell has found, a different path has been chosen. There is only one boundary condition. "The electromagnetic field has to be in a perfect equilibrium (balance) with itself and its surrounding." And when an electromagnetic field interacts with a gravitational field, exactly the same boundary condition is required. That is the single only requirement. From this single requirement follows one single equation. Eqn. (5) (gravity excluded) and eqn. (5a) (gravity included) in this manuscript.

All the solutions for electromagnetic configurations that are solutions of the Maxwell Equations are also solutions of eqn. (5). But because of the special mathematical structure of (5) other electromagnetic field configurations are possible. And this will have an impact on our understanding of the universe. Because we cannot understand the universe until we understand light [15-18].

Wheeler [1] introduced in 1953 the concept of GEONS (Gravitational ElectrO-magnetic eNtities) in which electromagnetic radiation has been confined by its own gravitational field. To calculate the dimensions of these gravitational-electromagnetic confinements Wheeler based his calculations on the Einstein-Maxwell equations, the mathematical ground on which the Theory of General Relativity has been built and found electromagnetic-gravitational confinements with a diameter of several lightyears and a lifetime of several milliseconds. The results were very disappointing because an elementary particle with a diameter of several lightyears and a lifetime of a few milliseconds can hardly be considered as an elementary particle (Figure 1).

In the presented theory the electromagnetic-gravitational interaction has been grounded on Newton's third law. It has been grounded on the stability of electromagnetic-gravitational fields in a perfect equilibrium with itself and its surrounding. In Table 1, electromagnetic-gravitational confinements have been presented for any harmonic (sinusoidal) frequency with an infinite lifetime and diameters varying from $<10^{-40}$ and $>10^{+40}[\mathrm{~m}]$ based on eqn. (5a) in a spherical coordinate system $[19,20]$.

Eqns. (5) and (5a) are presented in a coordinate-free system which makes it possible to change in a single step from cartesianinto spherical- and toroidal coordinates. Paragraph 2 represents the calculations that result into the Dynamic Equilibrium eqns. (5) and (5a). Because the eqns. (5) and (5a) are new equations which have never been published before, evidence is required that these equations fit into the regular well known physics in the Electromagnetic Field

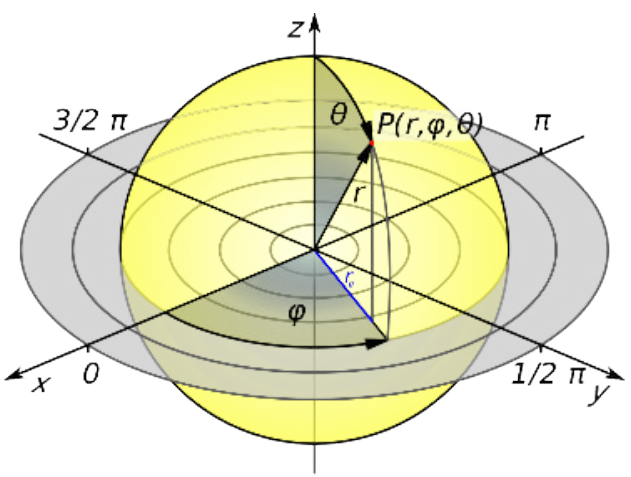

Figure 1: Electromagnetic radiation within a spherical coordinate.
Theory, presented by James Clerk Maxwell and the General Theory of Relativity, presented by Albert Einstein. Paragraph 3 presents this evidence in Cartesian Coordinates. Paragraph 3.1 describes in Cartesian Coordinates the well-known theory of Electromagnetism and paragraph 3.2 describes in a Cartesian Coordinate system an application in the General Theory of Relativity. Paragraph 4 presents this evidence in a Spherical Coordinate System.

Paragraph 5 presents an application of electromagneticgravitational confinements in a spherical Coordinate System in the General Theory of Relativity. Because the fundamental approach in electromagnetic field configurations is different than the classical approach by Maxwell and Einstein, a different result is expected. Instead of electromagnetic-gravitational confinements with a diameter of several lightyears and a lifetime of several milliseconds, presented by Wheeler in 1953, electromagnetic-gravitational confinements have been presented in Table 1 with diameters varying from $<10^{-40}[\mathrm{~m}]$ and $>10^{+40}[\mathrm{~m}]$ and an infinite lifetime for any harmonic frequency based on equation $(5 \mathrm{a})$.

Paragraph 6 discusses the possibilities of toroidal electromagneticgravitational confinements in a toroidal coordinates system.

\section{The Dynamic Equilibrium Equation}

In a way comparable to the way that GEONS (Gravitational ElectrO-magnetic eNtities) are described by Wheeler in General Relativity by the Gravitational-Electromagnetic Equilibrium Equation (the Einstein-Maxwell Equations), Electromagnetic Confinements are described by the Dynamic Equilibrium eqn. (5).

The Maxwell tensor equals:

$$
T^{a b}=\frac{1}{\mu_{0}}\left[F_{a c} F^{c b}+\frac{1}{4} \delta_{a b} F_{c d} F^{c d}\right]
$$

In which $F_{a b}$ are the elements of the Maxwell tensor defined by:

$$
F_{a b}=\partial_{b} \phi_{a}-\partial_{a} \phi_{b}
$$

The four-vector potential $\varphi_{a}$ is defined by: $\varphi_{a}=i \varphi / c, \vec{A}$ where $\varphi$ is the electric scalar potential, c the speed of light in vacuum and $\vec{A}$ is the magnetic vector potential. Substituting eqn. (2) in eqn. (1) results in the Energy Momentum Tensor:

$$
T^{a b}=\left[\begin{array}{rrrr}
w & -\frac{i}{c} S_{x} & -\frac{i}{c} S_{y} & -\frac{i}{c} S_{z} \\
& \varepsilon_{0}\left(E_{x}\right)^{2}+\mu_{0}\left(H_{x}\right)^{2}-w & \varepsilon_{0} E_{x} E_{y}+\mu_{0} H_{x} H_{y} & \varepsilon_{0} E_{x} E_{z}+\mu_{0} H_{x} H_{z} \\
-\frac{i}{c} S_{x} & & & \\
-\frac{i}{c} S_{y} & \varepsilon_{0} E_{y} E_{x}+\mu_{0} H_{y} H_{x} & \varepsilon_{0}\left(E_{y}\right)^{2}+\mu_{0}\left(H_{y}\right)^{2}-w & \varepsilon_{0} E_{y} E_{z}+\mu_{0} H_{y} H z \\
-\frac{i}{c} S_{z} & \varepsilon_{0} E_{z} E_{x}+\mu_{0} H_{z} H_{x} & \varepsilon_{0} E_{z} E_{x y}+\mu_{0} H_{z} H_{y} & \varepsilon_{0}\left(E_{z}\right)^{2}+\mu_{0}\left(H_{z}\right)^{2}-w
\end{array}\right]
$$

The force density $f^{a}$ in the 3 directions of the 3 coordinates of the chosen 3-coordinate system follows from the divergence of the electromagnetic energy-momentum tensor (3). Eqn. (4) gives the 3 -dimensional force density $f^{a}$ is a coordinate-free vector equation:

$$
-\frac{1}{c^{2}} \frac{\partial(\vec{E} \times \vec{H})}{\partial t}+\varepsilon_{0} \vec{E}(\nabla \cdot \vec{E})-\vec{\varepsilon}_{0} E \times(\nabla \times \vec{E})+\mu_{0} \vec{H}(\nabla \cdot \vec{H})-\mu_{0} \overrightarrow{\mathrm{H}} \times(\nabla \times \vec{H})=\vec{f}
$$

It has been assumed within the scope of this article that for any physical possible electromagnetic configuration (free radiation or confinement) it is the only required and sufficient boundary condition that the force densities in the 3 dimensions of the chosen coordinate system equal zero at any space at any time.

$$
\frac{\partial(\vec{E} \times \vec{H})}{\partial t}+\varepsilon_{0} \overrightarrow{\mathrm{E}}(\nabla \cdot \overrightarrow{\mathrm{E}})-\varepsilon_{0} \overrightarrow{\mathrm{E}} \times(\nabla \times \overrightarrow{\mathrm{E}})+\mu_{0} \vec{H}(\nabla \cdot \vec{H})-\mu_{0} \vec{H} \times(\nabla \times \vec{H})=\overrightarrow{0}
$$


An electromagnetic field which is in a perfect equilibrium with itself and its surrounding at any space and time, fulfills the necessary requirements for the physical possibility of the existence of this field. Under that condition eqn. (4) transforms into the Dynamic Equilibrium eqn. (5), which expresses the force density equals the vector zero of an electromagnetic field on itself and its surrounding in a perfect equilibrium. The part of the Dynamic Equilibrium Equation (DEE) (5), representing the equilibrium for the force densities in the 3 directions of the chosen coordinate system equals: The resulting REM equation equals:

The RGEM equation within a gravitational field $\vec{g}$ equals:

$$
\begin{aligned}
& -\frac{1}{c^{2}} \frac{\partial(\vec{E} \times \vec{H})}{\partial t}+\varepsilon_{0} \overrightarrow{\mathrm{E}}(\nabla \cdot \overrightarrow{\mathrm{E}})-\varepsilon_{0} \overrightarrow{\mathrm{E}} \times(\nabla \times \overrightarrow{\mathrm{E}})+\mu_{0} \vec{H}(\nabla \bullet \vec{H})-\mu_{0} \vec{H} \times(\nabla \times \vec{H}) \\
& -\frac{1}{2} \varepsilon_{0}^{2} \mu_{0}(\overrightarrow{\mathrm{E}} \bullet \overrightarrow{\mathrm{E}}) \overrightarrow{\mathrm{g}}-\frac{1}{2} \varepsilon_{0} \mu_{0}^{2}(\vec{H} \bullet \vec{H}) \overrightarrow{\mathrm{g}}=\overrightarrow{0}
\end{aligned}
$$

\section{EM Radiation within a Cartesian Coordinate System in the Absence of Gravity}

$$
\left(\begin{array}{c}
e_{x} \\
e_{y} \\
e_{z}
\end{array}\right)=\left(f(x, y) g\left(t-\left(\frac{\mathrm{K}_{1}}{z}+1\right) z \sqrt{\varepsilon_{0} \mu_{0}}\right)\right)
$$

The required Electromagnetic Field Configuration for a perfect Equilibrium in Space and Time follows from the dynamic equilibrium eqn. (5) and equals in Cartesian Coordinates $\{x, y, z, t\}$ for the Electric Field Components $\{x, y, z, t\}$ :

The required Electromagnetic Field Configuration for a perfect Equilibrium in Space and Time follows from the dynamic equilibrium eqn. (5) and equals in Cartesian Coordinates $\{x, y, z, t\}$ for the Magnetic Field Components $\{x, y, z, t\}$ :

$$
\left(\begin{array}{l}
m_{x} \\
m_{y} \\
m_{z}
\end{array}\right)=\sqrt{\frac{\varepsilon_{0}}{\mu_{0}}}\left(f(x, y) g\left(t-\left(\frac{\mathrm{K}_{1}}{z}+1\right) z \sqrt{\varepsilon_{0} \mu_{0}}\right)\right)
$$

In which $K_{1}$ is an arbitrary constant. For the divergence-free function $f(x, y)=1$, the solutions eqns. (6) and (7) are also the solutions for the known Maxwell Equations (Figure 2). For the non-divergencefree functions $f(x, y)$, the solutions (6) and (7) are not solutions for the Maxwell Equations, which requires divergence-free electromagnetic waves, propagating with the speed of light $c=1 / \sqrt{\varepsilon_{0} \mu_{0}}$, in the absence of any matter. But they are solutions of the Dynamic Equilibrium eqn.

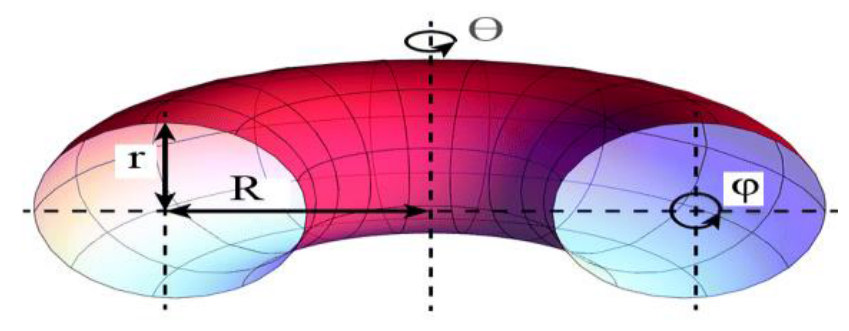

Figure 2: Radiation within a toroidal coordinate system.
(5) and clearly do exist in physics. Comparable with the projection of a slide with a beamer on a flat screen in the z-direction. In which the slide has an arbitrary intensity-division $f(x, y)$. The information $f(x, y)$ on the slide propagates with the speed of light $c=1 / \sqrt{\varepsilon_{0} \mu_{0}}$ towards the screen in the $\mathrm{z}$-direction in this example.

\section{EM Radiation within a Cartesian coordinate system under} the influence of a longitudinal gravitational field g:

The required Electromagnetic Field Configuration for a perfect Equilibrium in Space and Time for a Longitudinal Gravitational Field (The Light propagates in the same $\mathrm{z}$-direction as the $\mathrm{z}$-direction of the Gravitational Field) follows from the Dynamic Equilibrium eqn. (5a) and equals in Cartesian Coordinates $\{x, y, z, t\}$ for a gravitational field "g" for the Electric Field Components e $(x, y, z, t)$ :

$$
\left(\begin{array}{l}
e_{x} \\
e_{y} \\
e_{z}
\end{array}\right)=\left(\begin{array}{cc}
\mathrm{e}^{-\frac{1}{2} \mathrm{~g} \varepsilon_{0} \mu_{0} \mathrm{z}} & f(x, y) g\left(t-z \sqrt{\varepsilon_{0} \mu_{0}}\right) \\
0 \\
0
\end{array}\right)
$$

The required Electromagnetic Field Configuration for a perfect Equilibrium in Space and Time for a Longitudinal Gravitational Field (The Light propagates in the same $\mathrm{z}$-direction as the $\mathrm{z}$-direction of the Gravitational Field) follows from the Dynamic Equilibrium eqn. (5a) and equals in Cartesian Coordinates $\{x, y, z, t\}$ for a gravitational field "g" for the Magnetic Field Components $(x, y, z, t)$ :

$$
\left(\begin{array}{l}
m_{x} \\
m_{y} \\
m_{z}
\end{array}\right)=\sqrt{\frac{\varepsilon_{0}}{\mu_{0}}}\left(\begin{array}{c}
\mathrm{e}^{-\frac{1}{2} \mathrm{~g} \varepsilon_{0} \mu_{0} \mathrm{z}} \\
f(x, y) g\left(t-z \sqrt{\varepsilon_{0} \mu_{0}}\right) \\
0
\end{array}\right)
$$

Eqn. (6a) and (7a) are solutions of eqn. (5a) under the influence of a Longitudinal Gravitational field with field intensity "g" that acts along the $\mathrm{z}$-direction while the electromagnetic wave is also propagating in the $\mathrm{z}$-direction. The electromagnetic wave is propagating with the unaltered speed of light $c=1 / \sqrt{\varepsilon_{0} \mu_{0}}$, independently of the strength $\mathrm{g}$ of the gravitational field in the $\mathrm{z}$-direction. However, the amplitude of the electromagnetic wave becomes dependently of the gravitational intensity "g" and the distance " $\mathrm{z}$ " and changes along the $\mathrm{z}$-axis with the electromagnetic-gravitational interaction term $\mathrm{e}^{-\frac{1}{2} \mathrm{~g} \varepsilon_{0} \mu_{0} \mathrm{z}}$.

In this example is chosen for e.g. a laser beam positioned vertically on the ground on earth, shining vertically against the gravitational field "g" of the earth. Because the laser beam presents electromagnetic energy, the beam has electromagnetic mass. The potential energy of the electromagnetic mass is increasing while the laser light is propagating upwards, against the direction of the gravitational field. Because of the law of conservation of Energy, the electromagnetic energy is decreasing over a distance " $\mathrm{z}$ " proportional with the same amount $\mathrm{e}^{-\mathrm{g} \varepsilon_{0} \mu_{0} \mathrm{z}}$ as the potential energy of the electromagnetic mass is increasing.

\section{Electromagnetic Radiation within a Spherical Coordinate System}

The Spherical Coordinate System $\{r, \theta, \varphi, t\}$ is parameterized by the radius $r$ of the Sphere, the polar angle $\theta$ and the azimuthal angle $\varphi$ and the time $t$. 
The required Electromagnetic Field Configuration for a perfect Equilibrium in Space and Time follows from eqn. (5) and equals in Spherical Coordinates $\{r, \theta, \varphi, t\}$ for the Electric Field Components e $(\theta$, $r, \varphi, t)$ :

$$
\left(\begin{array}{l}
e_{r} \\
e_{\theta} \\
e_{\varphi}
\end{array}\right)=\left(\begin{array}{c}
0 \\
\frac{1}{r} f(\theta, \varphi) g\left(t-\left(\frac{\mathrm{K}_{1}}{r}+1\right) r \sqrt{\varepsilon_{0} \mu_{0}}\right) \\
0
\end{array}\right)
$$

The required Electromagnetic Field Configuration for a perfect Equilibrium in Space and Time follows from eqn. (4) and equals in Spherical Coordinates $\{r, \theta, \varphi, t\}$ for the Magnetic Field Components $\mathrm{m}(\theta$, $r, \varphi, t)$ :

$$
\left(\begin{array}{l}
m_{r} \\
m_{\theta} \\
m_{\varphi}
\end{array}\right)=\sqrt{\frac{\varepsilon_{0}}{\mu_{o}}}\left(\begin{array}{c}
0 \\
0 \\
\frac{1}{r} f(\theta, \varphi) g\left(t-\left(\frac{\mathrm{K}_{1}}{r}+1\right) r \sqrt{\varepsilon_{0} \mu_{0}}\right)
\end{array}\right) z z
$$

For the divergence-free function $f(\theta, \varphi)=1$, the solutions eqns. (8) and (9) are also the solutions for the known Maxwell Equations. For the non-divergence-free functions $f(\theta, \varphi)$, the solutions eqns. (8) and (9) are no solutions for the Maxwell Equations, which require divergence-free electromagnetic waves in the absence of any matter. They are however solutions of the DEE (5) and clearly they do exist in physics. Like the radiation of an inhomogeneous point light source like a LED.

\section{Confined Electromagnetic Radiation within a Spherical Coordinate System through Electromagnetic- Gravitational Interaction}

In physics it has been in generally assumed that the speed of light $c=1 / \sqrt{\varepsilon_{0} \mu_{0}}$ is a physical constant. In this paragraph the possibilities will be discussed of a variable speed of light that can vary from zero until values higher than $c$. The only requirement for the existence of an Electromagnetic Field Configuration will be the requirement of a perfect equilibrium in space-time for the chosen electromagnetic field configuration. This single unique requirement will always be a solution of the DEE (5).

The required Electromagnetic Field Configuration for a perfect Equilibrium in Space and Time in respectively the: $\theta$-direction $\left(f_{\theta}=0\right)$ and the $\varphi$-direction $(f \varphi=0)$ follows from eqn. (5). In Spherical Coordinates $\{r, \theta, \varphi, t\}$ the solution for the DEE (5) for the Electric Field Components $e\{\theta, r, \varphi, t\}$ equals:

$$
\left(\begin{array}{c}
e_{r} \\
e_{\theta} \\
e_{\varphi}
\end{array}\right)=\left(\begin{array}{c}
0 \\
f(r) g(\theta) h(\varphi) \operatorname{Sin}(\omega t) \\
-f(r) g(\theta) h(\varphi) \operatorname{Cos}(\omega t)
\end{array}\right)
$$

In Spherical Coordinates $\{r, \theta, \varphi, t\}$ the solution for the DEE (5) for the Magnetic Field Components $m\{\theta, r, \varphi, t\}$ in respectively the: $\theta$-direction $\left(f_{\theta}=0\right)$ and the $\varphi$-direction $(f \varphi=0)$ for the magnetic field components follows from eqn. (5) and equals:

$$
\left(\begin{array}{l}
m_{r} \\
m_{\theta} \\
m_{\varphi}
\end{array}\right)=\left(\begin{array}{c}
0 \\
f(r) g(\theta) h(\varphi) \operatorname{Cos}(\omega t) \\
f(r) g(\theta) h(\varphi) \operatorname{Sin}(\omega t)
\end{array}\right)
$$

Eqn. (4) gives the 3-dimensional force density $f^{a}$ of an ElectroMagnetic Field Configuration in a coordinate free vector equation. It follows from eqn. (4) that the radiation pressure in radial direction does not counterbalance and does not equal zero.

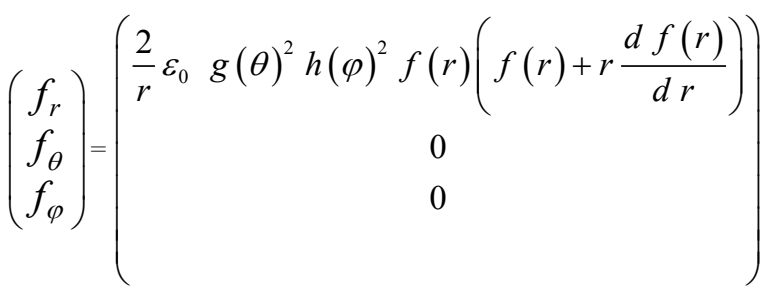

The energy-density $\mathrm{w}_{\mathrm{m}}$ of the Electromagnetic Configuration is essential for the calculation of the inward bounded gravitational pressure. The electromagnetic field configuration eqns. (10) and (11) for the functions $g(\theta)=1$ and $h(\varphi)=1$, results into the electromagnetic energy-density $\mathrm{w}_{\mathrm{em}}$ :

$$
\begin{aligned}
& \left(\begin{array}{l}
e_{r} \\
e_{\theta} \\
e_{\varphi}
\end{array}\right)=\left(\begin{array}{c}
0 \\
\mathrm{f}(\mathrm{r}) \operatorname{Sin}(\omega \mathrm{t}) \\
-\mathrm{f}(\mathrm{r}) \operatorname{Cos}(\omega \mathrm{t})
\end{array}\right) \quad\left(\begin{array}{l}
m_{r} \\
m_{\theta} \\
m_{\varphi}
\end{array}\right)=\left(\begin{array}{c}
0 \\
\mathrm{f}(\mathrm{r}) \operatorname{Cos}(\omega \mathrm{t}) \\
\mathrm{f}(\mathrm{r}) \operatorname{Sin}(\omega \mathrm{t})
\end{array}\right) \\
& \mathrm{w}_{\mathrm{em}}=\left(\frac{\mu_{0}}{2}(\overrightarrow{\mathrm{m}} \bullet \overrightarrow{\mathrm{m}})+\frac{\varepsilon_{0}}{2}(\overrightarrow{\mathrm{e}} \bullet \overrightarrow{\mathrm{e}})\right)=\varepsilon_{0} \mathrm{f}(r)^{2}
\end{aligned}
$$

According to Einstein's mass-energy equivalent $\mathrm{W}=\mathrm{mc}^{2}$, the specific electromagnetic mass [21-25]. Density $\rho_{\mathrm{em}}$ equals:

$$
\rho_{\mathrm{em}}=\frac{1}{\mathrm{c}^{2}}\left(\frac{\mu_{0}}{2}(\overrightarrow{\mathrm{m}} \bullet \overrightarrow{\mathrm{m}})+\frac{\varepsilon_{0}}{2}(\overrightarrow{\mathrm{e}} \bullet \overrightarrow{\mathrm{e}})\right)=\varepsilon_{0}^{2} \mu_{0} \mathrm{f}(\mathrm{r})^{2}
$$

The total electromagnetic mass $M_{e m}$ within a sphere with radius $R$ equals

$$
\mathrm{M}_{e m}=4 \pi \varepsilon_{0}{ }^{2} \mu_{0} \int_{0}^{\mathrm{R}} \mathrm{r}^{2} \mathrm{f}(\mathrm{r})^{2} \mathrm{dr}
$$

At a distance $r$ from the center of the sphere, the total electromagnetic mass $\mathrm{M}_{\mathrm{em}}$ within the sphere causes, according Newton's Shell Theorem a gravitational field [25-30].

Strength $g_{e m}$ :

$$
\mathrm{g}_{\mathrm{em}}=\frac{4}{\mathrm{r}^{2}} \pi \varepsilon_{0}^{2} \mu_{o} \mathrm{G}_{1} \int_{0}^{\mathrm{R}} \mathrm{r}^{2} \mathrm{f}(\mathrm{r})^{2} \mathrm{dr}
$$

In which $G_{1}$ is the gravitational constant and equals $G_{1}=6.67408$ $10^{-11}\left[\mathrm{~m}^{3} \mathrm{~kg}^{-1} \mathrm{~s}^{-2}\right]$. The gravitational inwards bounded radiation pressure [29-33] follows from eqn. (16):

$$
\mathrm{f}_{G R A V}=\rho_{\mathrm{em}} \mathrm{g}_{\mathrm{em}}=\frac{4}{\mathrm{r}^{2}} \pi \varepsilon_{0}^{4} \mu_{0}^{2} \mathrm{G}_{1} \mathrm{f}(r)^{2} \int_{0}^{\mathrm{R}} \mathrm{r}^{2} \mathrm{f}(\mathrm{r})^{2} \mathrm{dr}
$$

When there is a perfect equilibrium between the outwards bounded electromagnetic radiation pressure $f_{R A D}$ and the inward bounded gravitational pressure [31-38]. $f_{\text {GRAV }}$ we find from eqns. (12) and (17) the radius of the boundary sphere of the enclosed radiation $R_{\text {BOUNDARY }}$ for the functions $g(\theta)=1$ and $h(\varphi)=1$,

$$
f_{\text {RAD }}=\frac{2}{r} \varepsilon_{0} f(r)\left(f(r)+r \frac{d f(r)}{d r}\right)=\frac{4}{r^{2}} \pi \varepsilon_{0}^{4} \mu_{0}^{2} G_{1} f(r)^{2} \int r^{2} f(r)^{2} d r=f_{\text {GRA }}
$$

In the following example we choose for the function $f(r)$

$$
\mathrm{f}(\mathrm{r})=\mathrm{K}_{1} \mathrm{r}^{\mathrm{n}}
$$

Substituting eqn. (19) in eqn. (18) results in the equation for 
$\frac{8.019056 \times 10^{-94} \mathrm{~K}^{6} \mathrm{R}_{\text {BOUNDARY }}^{1+6 n}}{3+2 \mathrm{n}}=1.7708376 \times 10^{-11} \mathrm{~K}^{2}(1+\mathrm{n}) \mathrm{R}_{\text {BOUNDARY }}^{-1+2 n}$

The list below presents several values for the calculated Equilibrium Radius $R_{B O U N D A R Y}(2)$ at different values $\mathrm{K}_{1}$ and $\mathrm{n}$ :

\section{Confined Electromagnetic Radiation within a Toroidal Coordinate System}

The Toroidal Coordinate System $\{\theta, r, \varphi, R, t\}$ is parameterized by the large radius $\mathrm{R}$ of the torus. The Toroidal Coordinate System is obtained by rotating bipolar coordinates $\{r, \varphi\}$ around an axis perpendicular to the axis connecting the two foci. The coordinate $\{\theta\}$ specifies the angle of rotation. The torus in the Figure 3 below has been constructed with a Radius $\mathrm{R}=3$ and $\mathrm{r}=1$.

The required Electromagnetic Field Configuration for a perfect Equilibrium in Space and Time equals in Toroidal Coordinates $\{\theta, r, \varphi, t\}$ for the Electric Field Components e $(\theta, r, \varphi, t)$ :

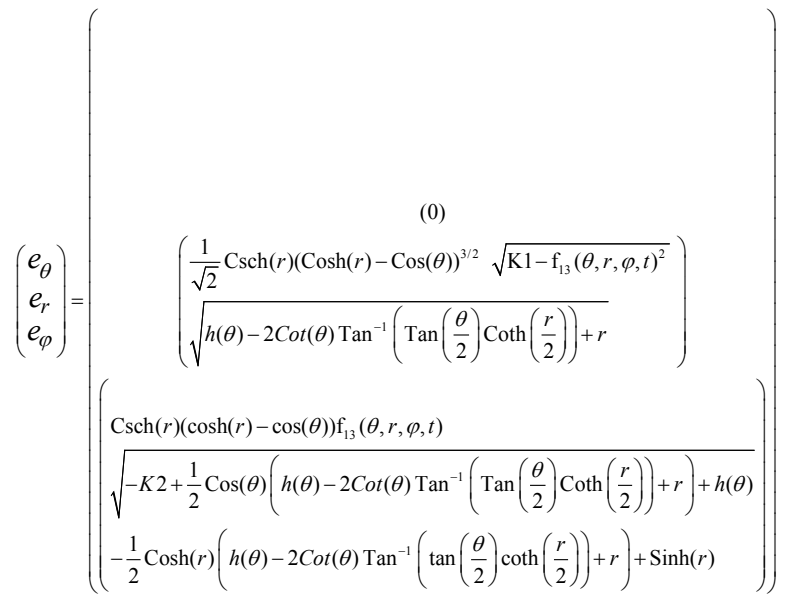

The required Electromagnetic Field Configuration for a perfect Equilibrium in Space and Time equals in Toroidal Coordinates $\{\theta, r, \varphi, t\}$ for the Magnetic Field Components $\mathrm{m}(\theta, r, \varphi, t)$ :

$$
\left(\begin{array}{l}
m_{\theta} \\
m_{r} \\
m_{\varphi}
\end{array}\right)=\sqrt{\frac{\varepsilon_{0}}{\mu_{0}}}\left[\begin{array}{c}
\left(\begin{array}{c}
\frac{-1}{\sqrt{2}} \operatorname{Csch}(r)(\operatorname{Cosh}(r)-\operatorname{Cos}(\theta))^{3 / 2} \mathrm{f}_{13}(\theta, r, \varphi, t) \\
\left.\sqrt{h(\theta)-2 \operatorname{Cot}(\theta) \operatorname{Tan}^{-1}\left(\operatorname{Tan}\left(\frac{\theta}{2}\right) \operatorname{Coth}\left(\frac{r}{2}\right)\right)+r}\right)
\end{array}\right. \\
\left(\begin{array}{l}
\operatorname{Csch}(r)(\cosh (r)-\cos (\theta)) \sqrt{\mathrm{K} 1-\mathrm{f}_{13}(\theta, r, \varphi, t)^{2}} \\
\sqrt{-K 2+\frac{1}{2} \operatorname{Cos}(\theta)\left(h(\theta)-2 \operatorname{Cot}(\theta) \operatorname{Tan}{ }^{-1}\left(\operatorname{Tan}\left(\frac{\theta}{2}\right) \operatorname{Coth}\left(\frac{r}{2}\right)\right)+r\right)+h(\theta)} \\
-\frac{1}{2} \operatorname{Cosh}(r)\left(h(\theta)-2 \operatorname{Cot}(\theta) \operatorname{Tan}^{-1}\left(\tan \left(\frac{\theta}{2}\right) \operatorname{coth}\left(\frac{r}{2}\right)\right)+r\right)+\operatorname{Sinh}(r)
\end{array}\right)
\end{array}\right]
$$

\section{Confined Electromagnetic Radiation within a Toroidal Coordinate System through Electromagnetic- Gravitational Interaction in a non-linear Space-Time Continuum}

The required Electromagnetic Field Configuration for a Gravitational-Electromagnetic Equilibrium in Space and

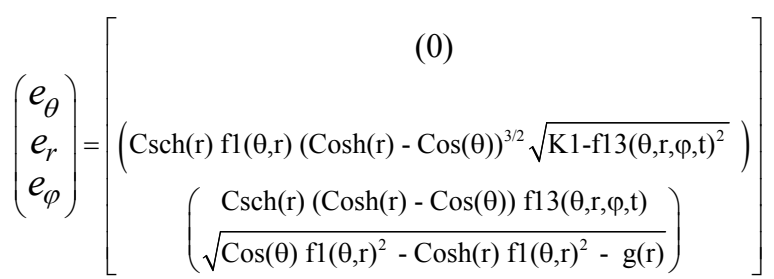

Time equals in Toroidal Coordinates $\{\theta, r, \varphi, t\}$ for the Electric Field Components $\mathrm{e}(\theta, r, \varphi, t)$

The required Electromagnetic Field Configuration for a Gravitational-Electromagnetic Equilibri12um in Space and Time equals in Toroidal Coordinates $\{\theta, r, \varphi, t\}$ for the Magnetic Field Components $\mathrm{m}(, r, \varphi, t)$ :

$$
\left(\begin{array}{l}
m_{\theta} \\
m_{r} \\
m_{\varphi}
\end{array}\right)=\sqrt{\frac{\varepsilon_{0}}{\mu_{0}}}\left[\begin{array}{c}
(0) \\
\left(\operatorname{Csch}(\mathrm{r}) \mathrm{f} 1(\theta, \mathrm{r})\left(-(\operatorname{Cosh}(\mathrm{r})-\operatorname{Cos}(\theta))^{32}\right) \mathrm{f} 13(\theta, \mathrm{r}, \varphi, \mathrm{t})\right.
\end{array}\right)
$$

The toroidal electromagnetic field configuration is in perfect equilibrium with itself and its surrounding in respectively the - and the $\varphi$-direction Figure 3 . There is a resulting electromagnetic outward bounding force density in the r-direction, $\vec{f}(\theta, r, \varphi, t)$ indicated as the outward bounding radiation pressure of the toroidal electromagnetic confinement.

$$
\vec{f}(\theta, r, \varphi, t)=\left[\begin{array}{c}
0 \\
-\frac{\varepsilon_{\mathrm{o}} \mathrm{K} \operatorname{Csch}^{2}(\mathrm{r})(\operatorname{Cos}(\theta)-\operatorname{Cosh}(\mathrm{r}))}{\left(4 \mathrm{f1}(\theta, \mathrm{r}) \mathrm{f} \mathrm{fl}^{\prime \prime \prime \prime}(\theta, \mathrm{r})(\operatorname{Cos}(\theta)-\operatorname{Cosh}(\mathrm{r}))-\mathrm{g}^{\prime}(\mathrm{r})\right)} \\
2 \mathrm{R} \\
0
\end{array}\right]
$$

This resulting outward bounding radiation pressure has to be compensated by the inward bounding gravitational force density, to create the required equilibrium by electromagnetic-gravitational interaction.

In a comparable way as in the example presented in spherical coordinates in eqns. (13) and (14), the electromagnetic mass-density from the energy density in the torus can be calculated. With these values the inward bounded gravitational radiation pressure can be

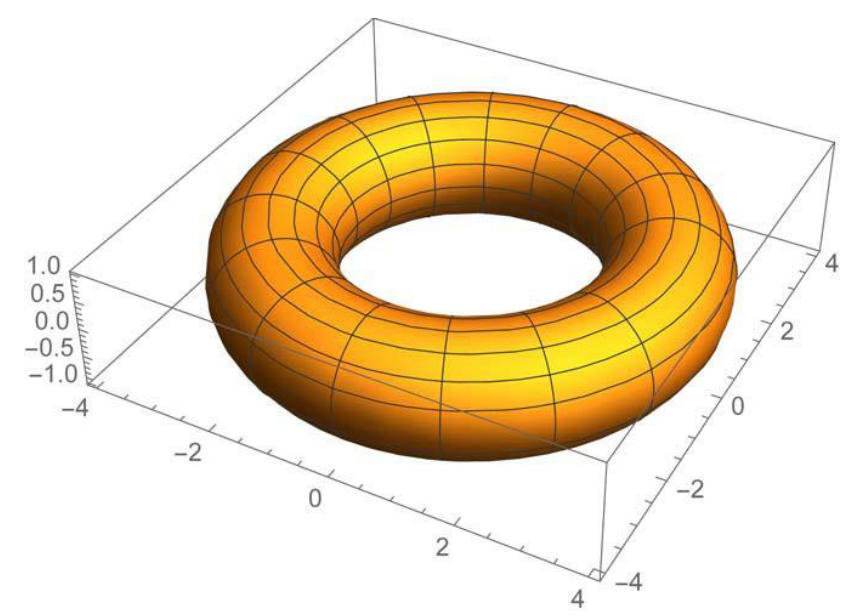

Figure 3: The torus constructed with a Radius $R=3$ and $r=1$. 
derived. From the requirement that both force densities have to compensate each other, the Boundary Radius $R_{B O U N D A R Y}$ for the toroidal confinement can be calculated.

\section{Concluding Remarks}

The example of Gravitational-Electromagnetic Interaction, presented in Table 1 shows two types of confinement.

1. For values $0<\mathrm{n}<-1$, the Gravitational-Electromagnetic Confinement will be Gravitationally controlled (Table 1). This means that for values for $r>R_{\text {BOUNDARY }}$ the inward bounded Gravitational for will be larger than the outward bounded Electromagnetic Radiation pressure. Electromagnetic Radiation will be attracted by Gravity towards the confinement at the surface $\mathrm{R}_{\text {BOUNDARY }}$. Because for values $\mathrm{r}<\mathrm{R}_{\text {BOUNDARY }}$ the outward bounded radiation pressure is higher than the inward bounded gravitational pressure, all the radiation will be forced to be confined at equilibrium just at the surface of the spherical sphere with radius $\mathrm{R}_{\text {BOUNDARY }}$. The confinement can be considered as an Electromagnetic Black Hole.

\begin{tabular}{|c|c|c|c|}
\hline Values for $n$ & Values for $K_{1}$ & $R_{\text {BOUNDARY }}[\mathrm{m}]$ & Area \\
\hline-10 & 1 & 0.000724227 & \multirow{8}{*}{$\begin{array}{c}\text { Electromagnetic Particle } \\
\text { (Electromagnetically } \\
\text { Controlled) }\end{array}$} \\
\hline-4 & 1 & $5.5940239 \times 10^{-10}$ & \\
\hline-2 & 1 & $6.7798267 \times 10^{-28}$ & \\
\hline-2 & 2 & $1.3559653 \times 10^{-27}$ & \\
\hline-2 & $10^{18}$ & $6.7798267 \times 10^{-10}$ & \\
\hline-1.6 & 1 & $3.0622921 \times 10^{-45}$ & \\
\hline-1.51 & 1 & $4.7888625 \times 10^{-52}$ & \\
\hline-1.51 & $10^{18}$ & $9.4264902 \times 10^{-17}$ & \\
\hline-1 & 1 & Infinite & Solution of (5) \\
\hline-0.9 & 1 & $1.2126075 \times 10^{267}$ & \multirow{4}{*}{$\begin{array}{c}\text { Electromagnetic Black } \\
\text { Hole (Gravitationally } \\
\text { Controlled) }\end{array}$} \\
\hline-0.5 & 1 & $2.1755186 \times 10^{54}$ & \\
\hline-0.5 & $10^{18}$ & $2.1755186 \times 10^{18}$ & \\
\hline-0.5 & $10^{36}$ & $2.1755186 \times 10^{-18}$ & \\
\hline
\end{tabular}

Table 1: Calculations of equilibrium radius $R_{\text {BOUNDARY }}(2)$ at different values $\mathrm{K}_{1}$ and $\mathrm{n}$.

2. For values $-1<\mathrm{n}<-\infty$, the Gravitational-Electromagnetic Confinement will be Electromagnetically controlled (Table 1). This means that for values for $r>R_{\text {BOUNDARY }}$ the inward bounded Gravitational for will be smaller than the outward bounded Electromagnetic Radiation pressure. Electromagnetic Radiation will be scattered by the Radiation Pressure away from the confinement at the surface $\mathrm{R}_{\text {BOUNDARY }}$. Because for values $r<R_{\text {BOUNDARY }}$ the outward bounded radiation pressure is smaller than the inward bounded gravitational pressure, all the radiation will be confined within the sphere with radius $R_{\text {BOUNDARY }}$. The confinement can be considered as an Electromagnetic Particle.

3. For values $n=-1$, the inward bounded Gravitational pressure equals the outward bounded Electromagnetic Radiation pressure at any distance $r$. The calculated value for $\mathrm{R}_{\text {BOUNDARY }}$ becomes $\mathrm{R}_{\text {BOUNDARY }} \rightarrow \infty$.

Because of the extremely high-energy densities within electromagnetic-gravitational confinements and the extremely small dimensions, the radiation pressure at small densities will be extremely high. For this reason, electromagnetic-gravitational confinements will behave like nonde formable particles in experiments.

\section{Acknowledgement}

This work has been made possible by the contributions of many. I am indebted to all and to my family, Lynn, Antonia and Julian in particular.

\section{References}

1. Wheeler J (1955) Geons. Phys Rev 97: 511.
2. Rosser WGV (2013) Classical Electromagnetism via relativity: An alternative approach to Maxwell's equations. Springer.

3. Molski M (1993) An Electromagnetic Approach to Special Relativity and Quantum Mechanics. Phys Essays 6: 143.

4. Jennison RC, Drinkwater AJ (1977) An approach to the understanding of inertia from the physics of the experimental method. J Phys A: Math Gen 13: 2247.

5. Jennison RC (1983) Wave mechanical inertia and the containment of fundamental particles of matter. J Phys A: Math Gen 16: 1-3635.

6. Jennison RC (1978) Relativistic phase-locked cavities as particle models. J Phys A: Mathand Gen.

7. Jehle H (1971) Relationship of Flux Quantization to Charge Quantization and the Electromagnetic Coupling Constant. Phys Rev D 3: 1-306.

8. Jehle H (1975) Flux quantization and fractional charges of quarks. Phys Rev D 11: 1-2147.

9. Jennison RC, Jennison MAC, Jennison TMC (1986) J Phys A: Math Gen 19 1-2249.

10. Molski M, Konarski J (1993) Extended Simons-Parr-Finlan approach to the analytical calculation of the rotational-vibrational energy of diatomic molecules. Physics Reviews A 47: 711.

11. Barut AO, Chandola HC (1992) "Localized" tachyonic wavelet solutions of the wave equation. Phys Lett A 180: 5-8.

12. Molski M (1991) Extended wave-particle description of longitudinal photons. J Phys A: Math Gen 24: 1-5063.

13. Elbaz C (1988) On self-field electromagnetic properties for extended material particles. J Phys Lett A 127: 308-314.

14. Elbaz C (1987) Optical properties of the Compton Effect. Phys A: Math Gen 20: $123-205$

15. Pauli W (1985) Wissenschaftlicher Briefwechsel mit Einstein, Bohr, Heisenberg Springer.

16. Bethe HA, Jackiw RW (1906-2005) Intermediate Quantum Mechanics. National Library of Australia.

17. Gottfried K, Weisskopf V (1986) Concepts of Particle Physics. Oxford University Press

18. Jehle H (1972) Flux Quantization and Particle Physics. Phys Rev D 6: 441.

19. Kaku M (1988) Introduction to Superstrings. Springer Verlag.

20. Freund $P(1986)$ Introduction to Supersymmetry. Cambridge University Press

21. Feynman R, Weinberg S (1986) Elementary Particles and the Laws of Physics Cambridge University Press, UK.

22. Casimir HBG (1953) Introductory remarks on quantum electrodynamics. Physica 19: 846.

23. Yaghjian AD (1989) Proceedings of the URSI International Symposium on Electromagnetic Theory. Int Union Radio Sci 14: 322.

24. Yaghjian AD (1977) A Direct Approach to the Derivadon of Electric Dyadic Green 's Functions. NBS TN 1000, Washington.

25. Power E, Wheeler J (1957) Thermal Geons. Rev Mod Phys 29: 480.

26. Petrich $\mathrm{W}$, Behavior of atoms in a compressed magneto-optical trap. J Opt Soc Am B 11: 1332.

27. Ovchinnikov YB (1994) Opt Spectrosc 76: 188.

28. Vegt JW (1995) A Continuous Model of Matter based on AEONs. Physics Essays 8: 201-224

29. Vegt JW (2002) The Maxwell-Schrödinger-Dirac correspondence in Auto Confined Electromagnetic Fields. Annals of the Louis de Broglie Foundation.

30. Vegt JW (1996) A particle Free Model of Matter based on Electromagnetic SelfCofinement. Annales Fondation Louis de Broglie.

31. Maldacena JM (1996) Black Holes in String Theory. Cornell University Library.

32. De Andrade VC, Pereira JG (1997) Gravitational Lorentz force and the description of the gravitational interaction. Phys Rev D 56: 468. 
33. Mohr PJ, Taylor BN, Newell DB (2008) CODATA recommended values of the fundamental physical contants 2006. Journal of Physical and Chemical Reference Data 37: 1187.

34. Hiscock WA (1985) Exact gravitational field of a string. Phys Rev D 31: 3288.

35. Degrand T, Jaffe L, Johnson K, Kiskis J (1975) Masses and other parameters of the light Hadrons. Physical Review D: Particles and Fields12: 2060-2076.
36. Krasnoholovets V (1997) Motion of a Relativistic Particle and the Vacuum Physics Essays 10: 407-416.

37. Jehle H (1972) Flux Quantization and particle Physics. Phys Rev D 6: 441-457.

38. Jehle H (1975) Flux Quantization and fractional charges of quarks. Phys Rev D 11: 2147. 